\title{
Morpho-physiological heterogeneity of cells within two rat prostate carcinoma cell lines AT-2 and MAT-LyLu differing in the degree of malignancy observed by cell cloning and the effects of caffeine, theophylline and papaverine upon a proportion of the clones
}

\author{
EWA MUSIALIK ${ }^{*}$, DAMIAN RYSZAWY* ${ }^{*}$ ZBIGNIEW MADEJA and WLODZIMIERZ KOROHODA \\ Department of Cell Biology, Faculty of Biochemistry, Biophysics and Biotechnology, \\ Jagiellonian University, 30-387 Krakow, Poland
}

Received November 6, 2012; Accepted January 8, 2013

DOI: $10.3892 /$ or.2013.2323

\begin{abstract}
Analysis of the heterogeneity of clones of single cells from two established lines of the Dunning series of rat prostate carcinoma differing in the degree of malignancy, AT-2 (moderately malignant) and MAT-LyLu (highly malignant), was conducted. The results showed that not only the original tumors and primary cell cultures were heterogeneous but also the established cell lines of tumor origin. In the MAT-LyLu cell line, the clones of cells with morpho-physiological features characteristic of malignant cells dominated, whereas diverse types of clones were present in the AT-2 cell line. Differences in cell morphology (EMT), multi-layering and release from contact inhibition followed by active migration were observed and found to be correlated with increased expression of proteins involved in cancer cell invasiveness: connexin 43 and transcription factor Snail. Caffeine, theophylline and papaverine, reported to show anticancer activity in vivo, were found to decrease the proportion of clones displaying malignant cell features in the AT- 2 cell line. At the tested concentrations, these compounds reversibly retarded cell growth but did not inhibit it. The results showed that the heterogeneity of cell populations within the cell lines should be taken into account in experiments carried out in vitro on established model cancer cell lines.
\end{abstract}

Correspondence to: Professor Wlodzimierz Korohoda or Professor Zbigniew Madeja, Department of Cell Biology, Faculty of Biochemistry, Biophysics and Biotechnology, Jagiellonian University, ul. Gronostajowa 7, 30-387 Krakow, Poland

E-mail:w.korohoda@uj.edu.pl

E-mail: z.madeja@uj.edu.pl

*Contributed equally

Key words: rat prostate carcinoma, clonal analysis, cell heterogeneity, caffeine, theophylline, papaverine, epithelial-to-mesenchymal transition

\section{Introduction}

It has been established that primary tumors and metastases are composed of subsets of diverse cell types (1-3), and the existence within tumors of a small fraction of cells representing cancer stem cells responsible for tumor recurrence after therapy has been postulated (4). On the other hand, in numerous biochemical and biological studies it is often assumed that the cells of established cell lines cultured in vitro represent relatively homogeneous cell populations. Cells of different lines are studied and the differences among them are correlated with the degree of malignancy (5-10).

The aims of the present study were: i) to examine the diversity of morpho-physiological cell features, which have often been correlated with cell invasiveness and malignancy (11-19) in two rat prostate carcinoma cell lines applying cell cloning; ii) to check the expression of connexin 43 and transcription factor Snail, involved in epithelial-to-mesenchymal transition (EMT) and cell invasiveness in morpho-physiologically different clones (20-23); and iii) to examine the effects of caffeine, theophyline and papaverine, recently reported to have anti-metastatic activity in vivo (24-27) upon proportions of the particular types of cell clones. Experiments were carried out on two rat prostate carcinoma cell lines of the Dunning R-3327 system, differing in their capacity to produce metastases. The AT- 2 cell line is characterized by moderate metastatic potential (approximately 5-20\%) whereas the malignant MAT-LyLu cell line has high metastatic potential (over 90\%) (5-7). These cell lines belong to a series of over 20 lines separated from the original Dunning R-3327 cell line, isolated from spontaneous rat prostate carcinoma in $1963(28,29)$.

\section{Materials and methods}

Cell culture. Cells of the AT-2 and MAT-LyLu cell lines were propagated in a standard humidified incubator at $37^{\circ} \mathrm{C}$ in $5 \%$ $\mathrm{CO}_{2} / 95 \%$ air atmosphere. Plastic culture dishes (plates and Petri dishes) were purchased from Falcon. Cells were grown in RPMI-1640 medium (Lonza) supplemented with 10\% heat- 

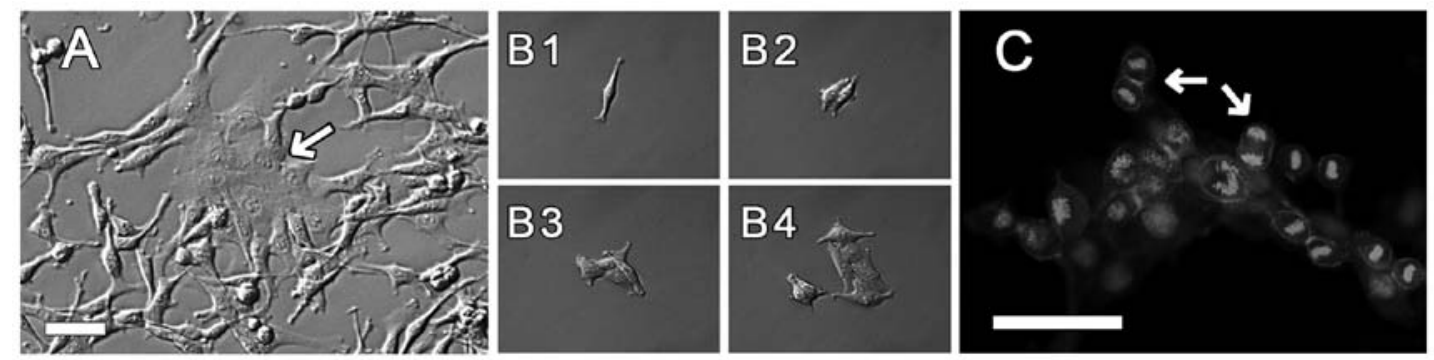

Figure 1. (A) High density culture of the AT-2 rat prostate carcinoma cell line exhibiting local differences in cell morphology as observed under phase contrast microscopy (objective, x20). The group of flattened, non-polarized cells is indicated with an arrow. (B1-B4) Frames from the time-lapse recording of a clone developing from a single AT-2 cell within $72 \mathrm{~h}$. (C) Synchronous cell divisions observed in a compact clone of flattened cells after $72 \mathrm{~h}$ of culture. The arrows point to cells in the anaphase state of mitosis. The bar corresponds to $50 \mu \mathrm{m}$.

inactivated fetal calf serum (Gibco BRL, Middlesex, UK) and a $1 \%$ antibiotic solution at a final concentration of $100 \mathrm{IU}$ penicillin, $100 \mu \mathrm{g}$ streptomycin, and $0.25 \mu \mathrm{g}$ amphotericin $\mathrm{B}$ per ml (Gibco BRL).

In cell cloning experiments, cells were seeded at a density of 300 cells per $6-\mathrm{cm}$ Petri dish (6 $\mathrm{cm}$ in diameter). After 2, 3, and 4 days the cells and their clones were observed under an inverted phase contrast microscope (objective, x20), and the particular types of clones were counted. Microphotographs were captured with a Leica DMI6000B inverted microscope with a DFC360FX CCD camera. For observations of cells incubated for longer than 5 days, particular clones were separated and transferred to 6-well plates. Cell viability was tested with trypan blue exclusion tests. Cell viability exceeded $90 \%$ in all experiments. Cells for the preparation of cell suspensions for seeding were counted using a Bürker hemocytometer.

Immunocytochemistry and fluorescence microscopy. Cells were plated on coverslips and cultured in RPMI-1640 medium for $72 \mathrm{~h}$. After washing in PBS, the cells were fixed for $10 \mathrm{~min}$ in $4 \%$ paraformaldehyde (PFA in PBS), and washed three times in PBS. The cells were then incubated for $10 \mathrm{~min}$ in $0.1 \%$ Triton X-100 permabilization solution, and washed three times in PBS. Cells were incubated in a blocking solution of 3\% BSA in PBS for $20 \mathrm{~min}$, double-stained for vinculin (Sigma) and F-actin (phalloidin conjugated with TRITC; Sigma) labeled with Alexa 488-conjugated goat anti-mouse IgG (Molecular Probes) and counterstained with Hoechst 333246 (Sigma). The cell staining was visualized using a Leica DMI6000B inverted microscope, and images were captured with a DFC360FX CCD camera.

Western blot analysis. Proteins from the cells were extracted with RIPA lysis buffer $(150 \mathrm{mM} \mathrm{NaCl}, 10 \mathrm{mM}$ Tris $\mathrm{pH} 7.5,1 \%$ NP4O, $1 \%$ deoxycholate, $0.1 \%$ SDS, protease inhibitor cocktail) (Roche). Proteins from total cell lysates were resolved on $10 \%$ SDS-PAGE gel, transferred to nitrocellulose membranes and blocked in 5\% non-fat milk in PBS/Tween-20. Blots were exposed to the primary rabbit polyclonal anti-Snail (1:500, Abcam), rabbit monoclonal anti-Cx43 (1:3000, Abcam), mouse monoclonal anti- $\alpha$-tubulin (1:3000, Sigma) and monoclonal mouse anti- $\beta$-catenin (1:3000, Santa Cruz) antibodies followed by detection of the antibodies using HRP-labeled secondary antibodies (1:3000, Invitrogen) and SuperSignal West Pico Substrate (Pierce, Rockford, IL). Visualization of the secondary antibody was performed using a chemiluminescence detection procedure according to the manufacturer's protocol (Microchemi). Tubulin was used as a loading control.

Time lapse-monitoring of the movement of individual cells. Cell movement was observed with a Leica DMI6000B inverted microscope with IMC contrast optics, and equipped with a digital DFC360FX CCD camera, at $37^{\circ} \mathrm{C}$ and in a $5 \%$ $\mathrm{CO}_{2}$ atmosphere. AT-2 clones were seeded into 6-well plates at a density of $4 \times 10^{2}$ cells $/ \mathrm{cm}^{2}$ and incubated in RPMI-1640 medium supplemented with 10\% FBS and antibiotics for $24 \mathrm{~h}$ before recording. The cell trajectories were constructed from 97 subsequent centroid positions recorded over $480 \mathrm{~min}$ at $5 \mathrm{~min}$ time intervals under a magnification of $x 20$. The cell trajectories were presented in circular diagrams with the starting point of each trajectory situated at the plot center (30-33). The Hiro program written by $\mathrm{W}$. Czapla was used for analysis of parameters characterizing cell locomotion, as previously described by Miekus et al (34). For each data point measured, at least 50 cells were analyzed.

\section{Results}

When cells grow to high densities in vitro, differences in cell size and shape are observed among groups of cells located in different regions of the single-cell culture vessel (35). In our experiments, several discrete cell phenotypes were noted within the same cultures. Fig. 1A shows an example of local groups of cells differing in morphology in AT-2 rat prostate cells grown in a single culture. It is possible to distinguish flat and well-spread epithelial-like cells among the dominant elongated, spindle-like cells. It is difficult to analyze such local differences in crowded cell cultures. Therefore, we observed cell clones originating from single cells. Fig. 1B1-B4 shows the development of a cell clone from one cell as monitored for 3 days with time-lapse photography. We noted (also in further experiments) that cell divisions within clones occurred synchronously, in particular in compact clones composed of cells (Fig. 1C). In some cases, as observed with time-lapse photography, almost all cells in clones composed of 8-32 cells were simultaneously undergoing mitosis.

In order to obtain further information on the morphological heterogeneity of AT-2 and MAT-LyLu rat prostate cancer cell lines in subsequent experiments, we examined clones grown under identical cell culture conditions. It was 

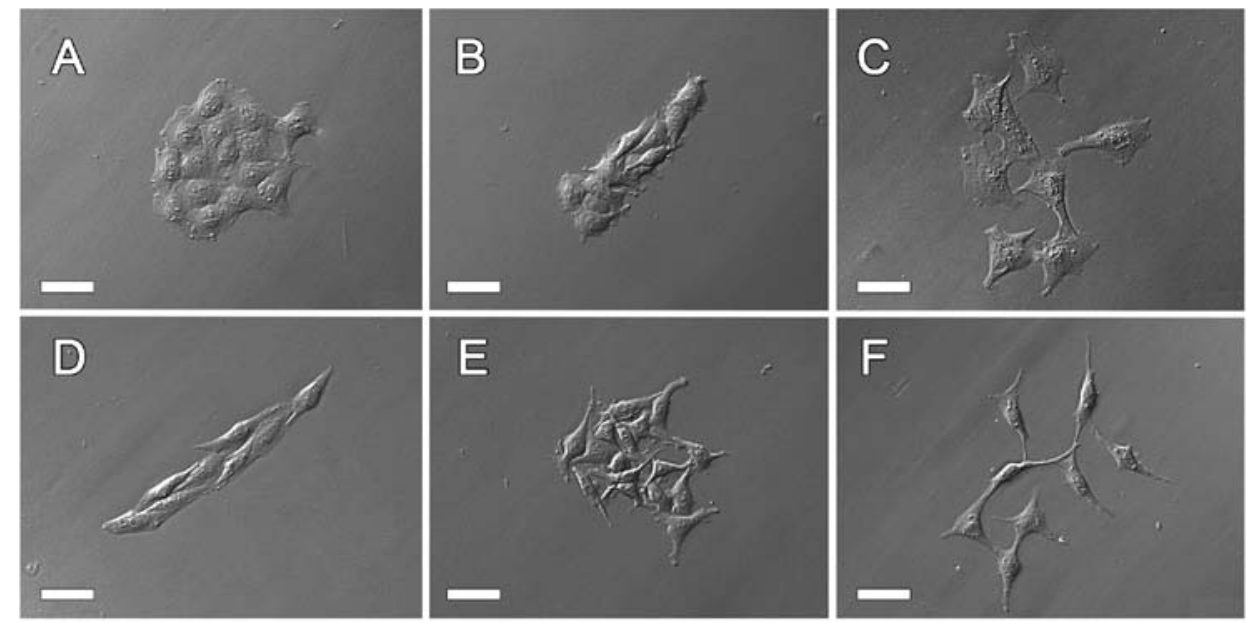

Figure 2. Six main types of clones developed from single cells isolated from an established AT-2 cell line of rat prostate carcinoma. For a more detailed explanation of the denotation of the clonal types E1, E2, E3 (epithelial-like) and M1, M2, and M3 (mesenchymal-like) as shown in A-F refer to the text. The bar corresponds to $50 \mu \mathrm{m}$.
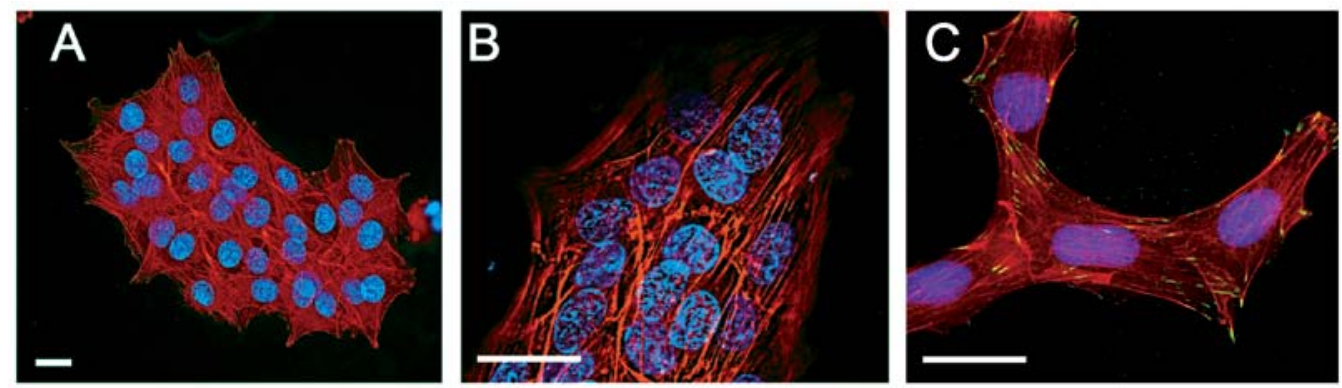

Figure 3. Fluorescence microscopic image of the occurrence of stress fibers, vinculin in cell-to-cell contacts and nuclei overlapping in multi-layered clones of the E2 and M3 clonal types of the AT-2 cell line. Actin cytoskeleton is stained red with TRITC-phalloidin, vinculin is stained green with Alexa 488 antibody and nuclei with Hoechst 333246. Bar corresponds to $25 \mu \mathrm{m}$.

difficult to discern morphological differences among single cells immediately after seeding. After the first 2-3 cell divisions, when small clones composed of 4-8 cells appeared, the diversity of cells and clones became distinguishable. After 3-6 days in culture, clones originating from single cells clearly differed among themselves in respect to cell characteristics which are known to correlate with neoplastic growth and/ or invasiveness. Analysis of cell morphology in the different clones limited to their description as epithelial or spindle-like (mesenchymal-like) appeared insufficient.

We observed three characteristic morpho-physiological features of cells and their clones: i) cell shape within clones: epithelial-like (unpolarized, well-spread) vs. mesenchymallike (spindle-like, elongated); ii) mode of cell multi-layering by formation of compact bumps (knots, bulges, domes) of flattened, non-polarized cells or by loosely packed layers of elongated cells crossing one another with their filopodia; and iii) clone organization, i.e. compact vs. dispersed with single cells emigrating rapidly from the clone. Three different types of epithelial-like cell clones were distinguished as well as the corresponding three clonal types of mesenchymal-like cells. The morphology of these six diverse types are shown in Fig. 2. The well-delineated compact clones of unpolarized, epithelial-like cells (designated as E1) or elongated, polarized, less spread and usually arranged in parallel mesenchymal-like cells (designated as M1) are shown in Fig. 2A and D. In these clones the cells grew in monolayers and exhibited contact inhibition of movement. E2 and M2 were designated as clones which were composed of well-delineated groups of epitheliallike (E2) or mesenchymal-like (M2) cells showing lack of contact inhibition and growing in multi-layers (Fig. 2B and E). E3 and M3 were colonies formed by cells of epithelial-like (E3) or mesenchymal-like (M3) morphology which immediately after divisions separated from one another and actively migrated as single cells (Fig. 2C and F). Clones E2 and M2 appeared more frequently on days 4-6, when the majority of individual clones contained $>20$ cells. Epithelial-like cells in E2 clones formed rather regular, multi-layered domes (bulbs), whereas elongated cells randomly crossed one another with their filopodial extensions in M2 clones (Figs. 3A and B; 4B and $\mathrm{C}$ ). These cell features were observed in compact clones originating from both AT-2 and MAT-LyLu cell lines in spite of their differences in malignancy, although compact clones were less frequent in the highly malignant MAT-LyLu cell line.

The morphology of E1, E2 and M1, M2 clones was additionally examined after fixation and staining of nuclei with Hoechst, the actin cytoskeleton with TRITC phalloidin, 

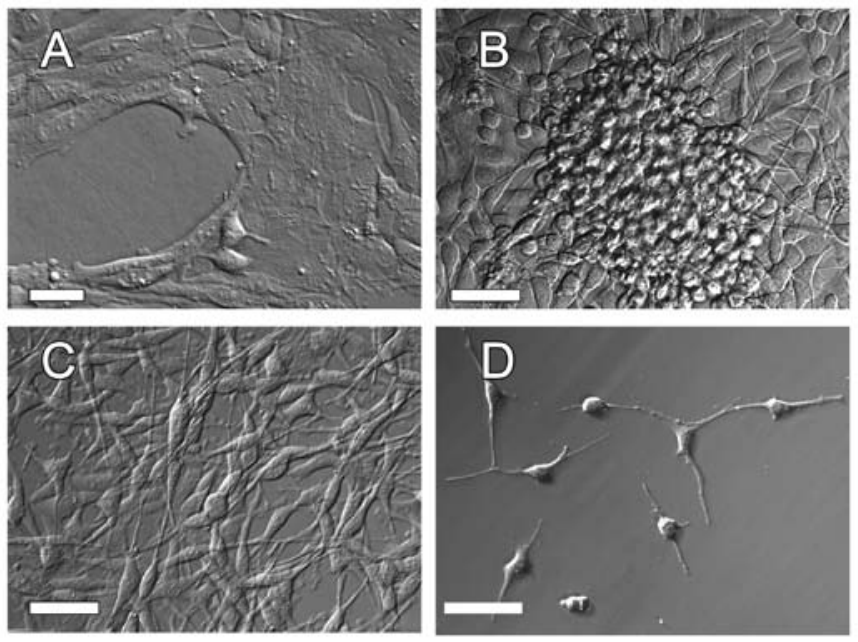

Figure 4. Maintenance of morpho-physiological cell features after subcultivation and expansion in the separated cultures for several days. (A) E1 clones and (B) E2 clones with local multi-layering and cells forming a bulge (node) (C) M2 clone with multi-layered cells released from contact inhibition and (D) arborized cells with long, thin filopodial extensions in subsets of AT-2 clones grown in the presence of papaverine for $96 \mathrm{~h}$. Bar corresponds to $50 \mu \mathrm{m}$.

and vinculin green with Alexa 488 (Fig. 3). This facilitated discrimination of cell multi-layering in the E2 and M2 clones. The visualization of the actin cytoskeleton revealed that in compact clones stress fibers were present even in cells of the upper layers and that vinculin occurs in cell-to-cell contacts (Fig. 3B and C).

The morpho-physiological features of the cells forming particular types of clones was preserved when the clones continued to grow in separation from one another as shown in Fig. 4A-C. This made it possible to analyze the selected protein expression using western blotting. Recently, the expression of connexin 43 and transcription factor Snail in cancer cells has been postulated to be involved in cancer cell invasiveness $(18-21,23)$. Western blot analyses of tubulin, $\beta$-catenin, connexin 43 and Snail expression in six diverse morpho-physiological types of clones of single AT-2 cells are illustrated in Fig. 5. High expression levels of connexin 43 and Snail were found in dispersed clones, in which cells rapidly separated from one another and showed active translocation, and in multi-layered clones, independent of whether they exhibited epithelial-like or mesenchymal-like morphologies.
The increased expression of connexin 43 was also observed in compact, monolayered clones of mesenchymal-like cells but not in compact epithelial-like clones. The expression of $\beta$-catenin was similar in all clone types.

Time-lapse analysis of the motility of the single cells separated from various clones confirmed that mesenchymallike cells from clones of corresponding morphology exhibited greatly increased motility. In particular, the greatest final displacements and lengths of cell trajectories of cells recorded $24 \mathrm{~h}$ after isolation from mesenchymal-like M3 and M2 clones as wells as from E3 epithelial-like clones are shown in circular diagrams in Fig. 6.

The distribution of different clones in cultures of AT-2 and MAT-LyLu cells revealed that in the AT-2 cell line more E1-3 clone types occurred whereas in the MAT-LyLu cell line (characterized by a high metastatic potential) M3 clone types dominated (Fig. 7A1 and B1). Under control conditions after $72 \mathrm{~h}$ in culture, $>90 \%$ of clones from both cell lines contained $>20$ cells. These results permitted further examination of the effects of caffeine, theophylline and papaverine, recently reported to influence the growth of tumors in vivo (24-27), upon growth and the proportions of cell clones with defined morphology in the rat prostate cancer cell lines. In preliminary experiments, we observed the effects of the tested substances on cell viability (data not shown). In subsequent experiments, concentrations were chosen which did not alter cell viability $>3 \%$ after 4 days of culture. The effects of a $2-\mathrm{mM}$ concentration of caffeine, 2-mM and 1.5-mM concentrations of theophylline, and a $0.2-\mathrm{mM}$ concentration of papaverine upon the proportion of various types of clones in AT-2 and MAT-LyLu cell lines grown for 3 days are shown in Fig. 7A2-A4 and B2-B4. These concentrations appeared to clearly influence the clonal growth of cells of the examined cell lines. Caffeine and theophylline had similar effects except that theophylline appeared to have a greater toxic effect on the MAT-LyLu than on AT-2 cells and a 2-mM concentration inhibited the formation of MAT-LyLu clones. Therefore, a $1.5-\mathrm{mM}$ concentration was used in this case. Papaverine, in addition to inducing changes in the proportion of various types of clones, caused visible changes in the morphology of the AT-2 cells. Numerous AT- 2 cells produced very long filopodia-like or dendrite-like extensions resembling those of neuronal cells (Figs. 4D and 7A4).

In AT- 2 cells, a clear decrease in the proportion of spindlelike cells (M) and E3 and M3 clones in relation to the compact clones (E1 and M1) was visible, and cell overlapping, at least

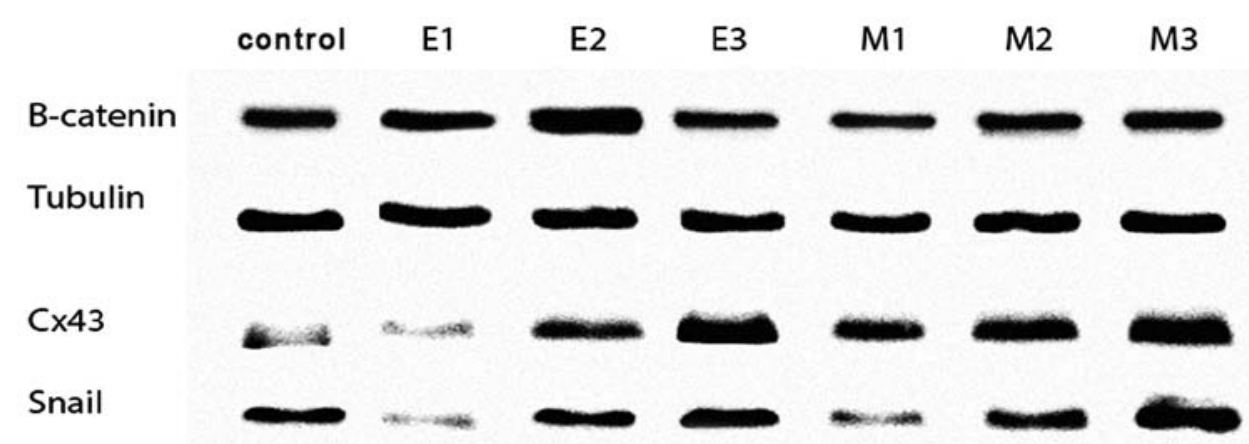

Figure 5. Western blot analysis of $\beta$-catenin, tubulin, connexin 43 (Cx43) and Snail proteins in cells from different morpho-physiological clones of diverse cell types and the control culture. Please note the increased expression of Snail and Cx43 in E3, M2, and M3 clones. 

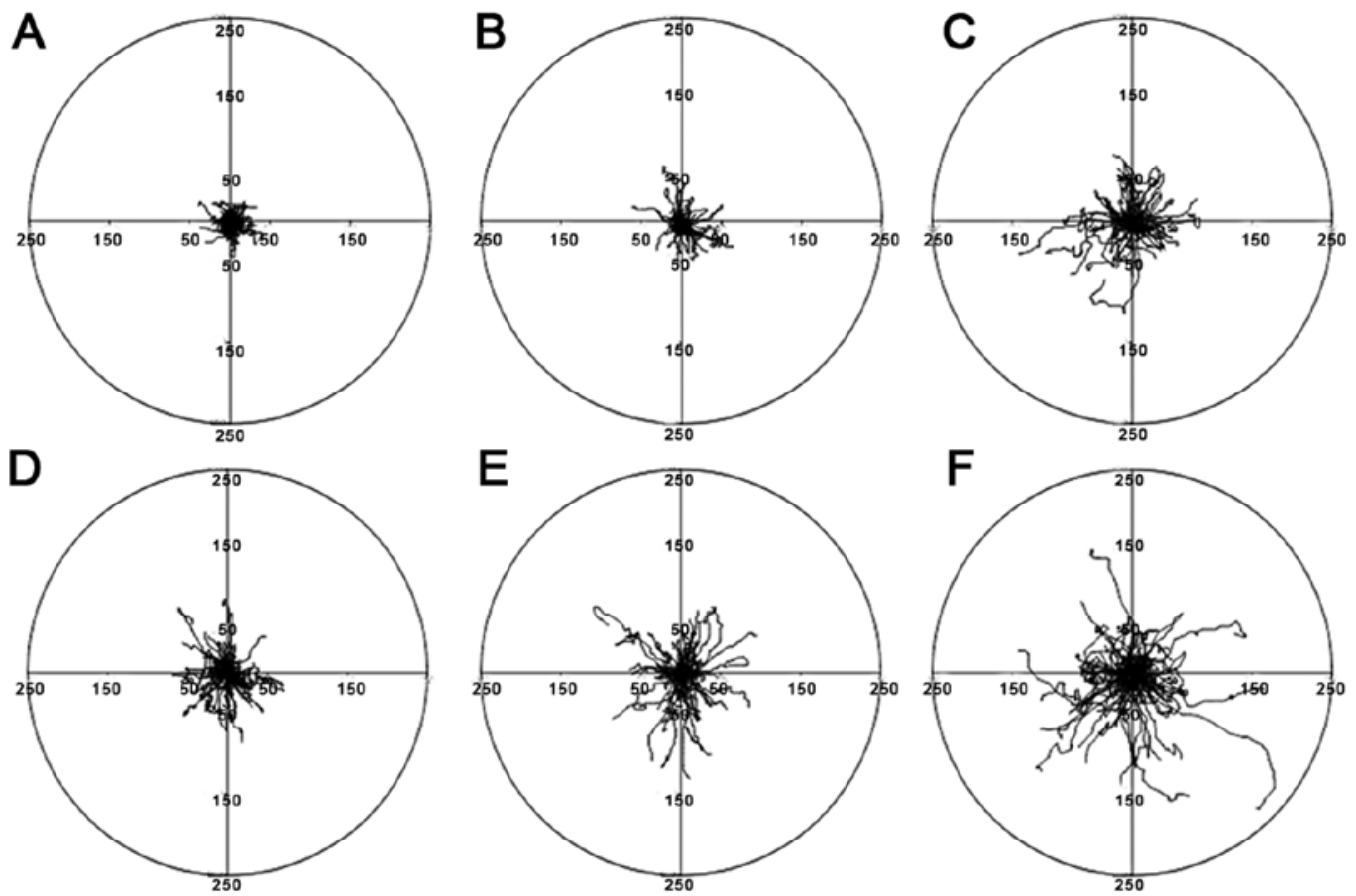

Figure 6. Circular diagrams showing the motility of cells isolated from the diverse clonal types and grown for $24 \mathrm{~h}$ prior to the determination of single-cell trajectories. Motility of cells from the (A) E1, (B) E2, (C) E3, (D) M1, (E) M2 and (F) M3 clones, respectively. Please note the increased motility of the cells isolated from the clones; $\mathrm{M} 3>\mathrm{M} 2>\mathrm{E} 3$.

within the first few days of culture, disappeared. These effects occurred in parallel with the cell growth retardation in the presence of $2 \mathrm{mM}$ caffeine and theophylline. Cell numbers in the single clones continuously increased although much more slowly than that under control conditions. After $72 \mathrm{~h}$, the number of single clones counted was usually 8 cells whereas under control conditions this number was $>20$. The retardation of cell growth, strongest in the presence of $0.2 \mathrm{mM}$ papaverine, was reversible (Fig. 8). Experiments in which the number of cells in the culture was estimated could not discriminate between the fast growth of only a subset of cells, or rather slight acceleration of growth of all cells present in the culture vessel. We could not, therefore, resolve whether the arborized cells with strongly modified morphology could revert to normal morphology and undertake growth or if these cells were rapidly overgrown by cells with spindle-like morphology. This aspect requires separate experiments.

\section{Discussion}

Permanent neoplastic cell lines often serve as models in the research of the biology of cancer cells. The Dunning series of rat prostate cancer cell lines have a common origin from a single tumor but are characterized by a different capacity to produce metastases when tested in vivo. The AT- 2 cell line is moderately malignant (producing less than $20 \%$ metastases) whereas the highly malignant MAT-LyLu cell line has the capacity to metastasize in more than $90 \%$ of cases (5-7). Our experiments revealed that both of these cell lines are heterogeneous, but the clones of single cells from the AT-2 line displayed a greater heterogeneity than the clones from the MAT-LyLu cell line.
Clonal analysis showed that the clones differed in regards to morpho-physiological cell features commonly correlated with neoplastic cell characteristics including invasiveness and capacity to metastasize. Typical features correlated with malignancy include: i) low stability of cell-to-cell contact, release from contact inhibition of growth and movement, resulting in cell multi-layering and random growth $(12,14,36)$; and ii) the transition from unpolarized, flat epithelial-like cell shapes to spindle-like, elongated mesenchymal-like shapes of invasive cells of high tumor-producing capacity $(11,36,37)$, which is usually described in the contemporary literature as EMT (16-19,22). The presence of these cell features was examined in clones of cells of the AT-2 and MAT-LyLu rat prostate adenocarcinoma cell lines. The main observation is that the clones of single cells from one line showed diverse expression of features commonly considered as characteristic for cancer cells. Apart from clones showing typical characteristics of malignant cells (designed as M2 and M3 clone types), clones resembling normal epithelial or mesenchymal cells were also present (i.e. E1 and M1 clones). These are compact clones of cells showing contact inhibition of movement and growth in one cell layer. In clones of the MAT-LyLu cell line, cells exhibiting typical features of highly malignant cells dominated. They grew and when dispersed, actively moved and separated from one another after division, assuming a spindle-like morphology or forming multi-layers of cells released from contact inhibition, crossing one another. The reported higher capacity of the MAT-LyLu cell line to form metastases in vivo than the AT-2 cell line (5-7) may be associated with a much higher proportion of M2 and M3 type clones in the former. The cells isolated from dispersed mesenchymal-like clones (M3) showed the highest motility 

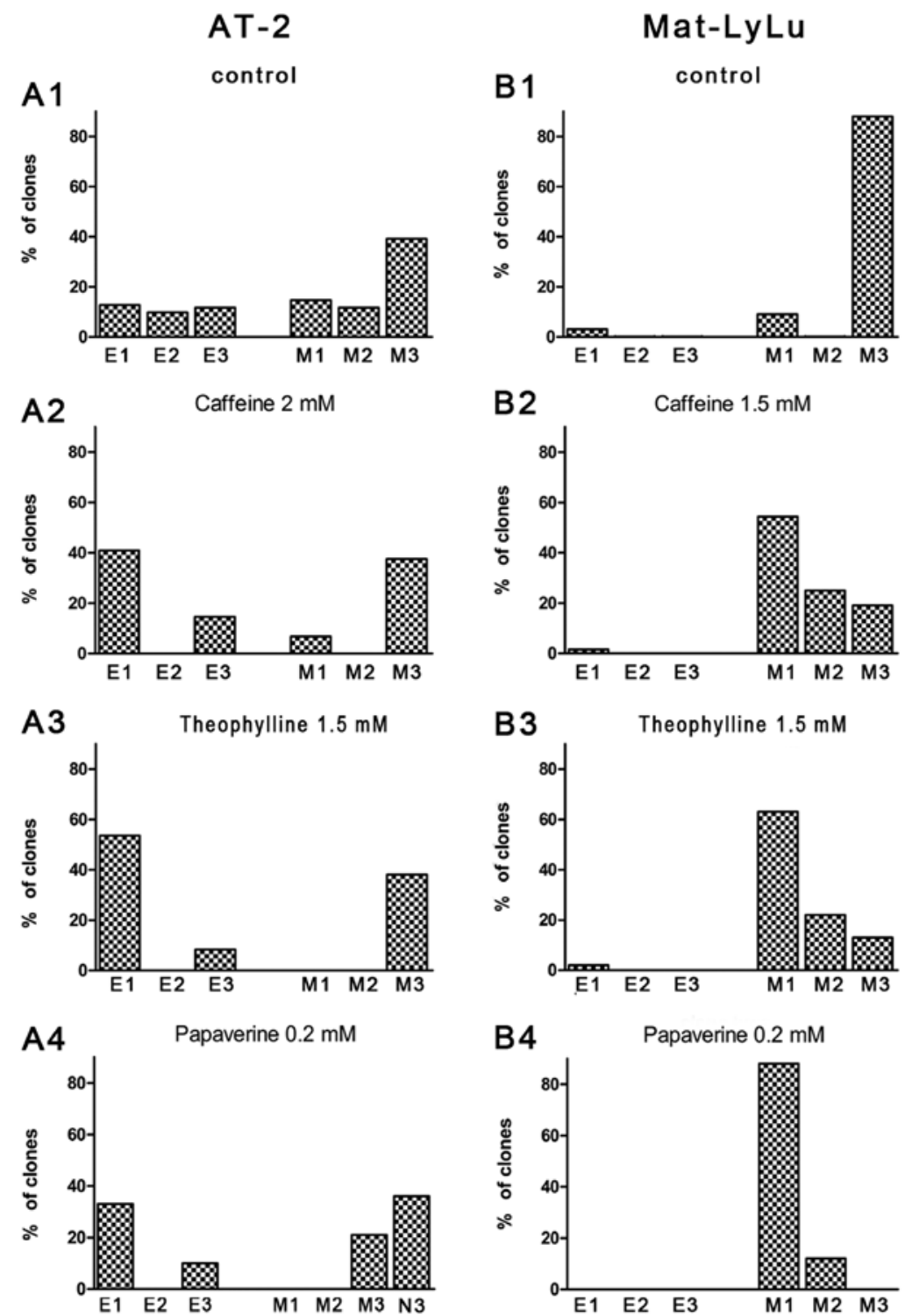

Figure 7. (A1 and B1) Distribution of diverse clonal types in the control cultures of cells from the AT-2 and Mat-LyLu rat carcinoma prostate cell lines. Please note the greater heterogeneity of clones from the AT-2 cell line than that from the MAT-LyLu cell line, in which dispersed M3 clones dominated. Effects of the presence of (A2 and B2) $2 \mathrm{mM}(1.5 \mathrm{mM}$ ) caffeine (A3 and B3) $2 \mathrm{mM}$ theophylline, and (A4 and B4) $0.2 \mathrm{mM}$ papaverine on the proportion of the diverse types of clones after $72 \mathrm{~h}$ of culture in the AT-2 (A2-A4) and MAT-LyLu (B2-B4) cells. E1-E3 and M1-M3 clones are described in Fig. 2 and N3 clones in the AT-2 cell line correspond to clones of arborized cells shown in Fig. 4D.
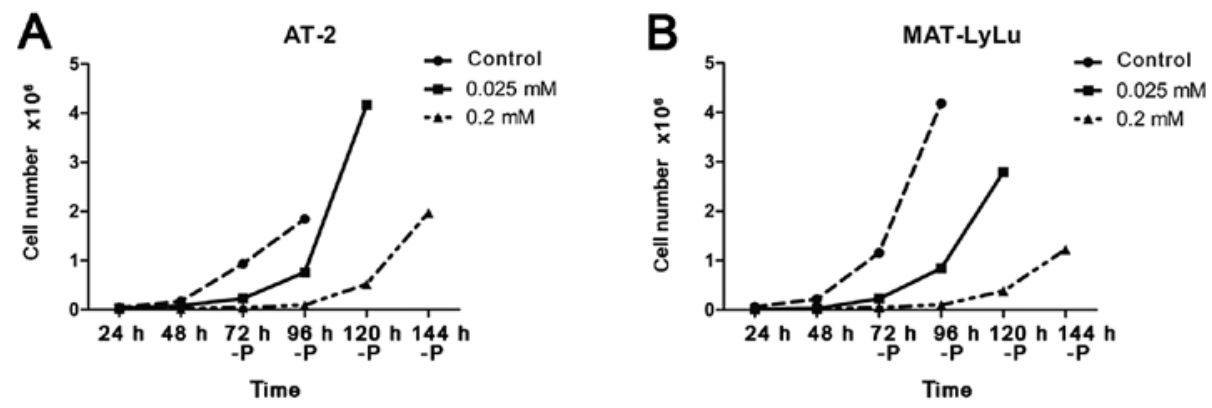

Figure 8. Retardation of AT-2 (A) and MAT-LyLu (B) cell growth in the presence of $0.2 \mathrm{mM}$ papaverine for $72 \mathrm{~h}$ in the culture medium and restoration of the rapid cell growth after removing of papaverine (-P) after $72 \mathrm{~h}$.

when compared with cells separated from the other clonal types. This is in accordance with the common postulate that highly invasive cells are characterized by high motile activity. In the AT- 2 cell line, cell clones of the malignant morpho-physiological phenotype were present, although in a much lower proportion. 
Markedly,in some cell clones of epithelial-like morphology, cells maintaining an epithelial-like shape (typically half-moonlike) could separate from one another and disperse showing active motility (cells from A3 clones). These results suggest that apart from the need to compare the properties among cells of different established cell lines it is advantageous to take into account the heterogeneity of cells within these cell lines. This may also include the molecular study of various proteins, the expression of which is postulated to be associated with cell malignancy, invasiveness and EMT. Recently, it was reported that the increased expression of connexin 43 and transcription factor Snail is associated with increased motility, invasiveness and EMT in prostate cancer cells $(18-21,23)$.

Our western blot analysis revealed that in accordance with these suggestions in the clones of single cells from the AT-2 line, a concomitant increase in the expression of connexin 43 and Snail was observed in dispersed clones of cells rapidly separated from one another showing active fast translocation, and in multi-layered clones, independent of whether the cells had epithelial-like or mesenchymal-like morphology. The increased expression of connexin 43 was also observed in compact, monolayered clones of mesenchymal-like cells but not in compact epithelial-like clones. The expression of $\beta$-catenin was similar in all clonal types. These results support the conclusion that the anti-proliferative activity of potential anticancer drugs should consider the effects of the tested compounds on particular cell types and their clones present within the established cancer cell lines propagated in vitro.

We examined the effect of three substances, caffeine, theophylline and papaverine, recently found to have limited anticancer activity in prostate cancer (24-27). In the AT-2 cell line, these agents decreased the proportion of clones having features attributed to malignancy at concentrations which reversibly retarded cell growth but did not inhibit cell multiplication and formation of clones (37). The results of observations performed in vitro correspond to reports showing moderate anticancer activity of caffeine and theophylline in vivo. In addition, papaverine strongly modified the shape of cells in some subsets of clones causing cell arborization and formation of long dendrite- or axon-like protrusions. Similar modifications of cell shape in the presence of papaverine were described in a fraction of mouse neuroblastoma cell culture (38). We suggest that differences in cell heterogeneity within cell lines apart from the differences among cell lines should be taken into account in experiments in which the effects of anticancer agents are examined using established cancer cell lines in vitro. Changes in the proportion of different clones should be considered in studies of the effects of agents modifying cell features. The presentation of cases in regards to the transition of morphology of single clones appears insufficient. Moreover, the promotion and progression in cancer cell lines expanded in vitro should be investigated with methods of single-cell cloning taking into account initial cell diversity.

Time-lapse recording of the development of clones from single cells revealed extensive synchronization of cell divisions maintained for a few cell generations in compact clones. This can provide a convenient model for research into the dependence of cell features and activity upon cell progression through phases of the cell cycle.
Our results emphasize the need for clonal analysis of cancer cell reactions to various factors, including anticancer agents, when experiments are carried out using established cancer cell lines in vitro. The diversity of cells within the propagated in vitro established cell lines may be associated with, and correspond to, the heterogeneity and genetic instability of cells within tumors in vivo and their primary cultures $(1-3,11,39)$. This implies that in addition to the differences among established cancer cell lines, differences concerning cells within cell lines should not be neglected when conducting research on the biology of cancer cells carried out under cell culture conditions.

\section{Acknowledgements}

This work ws financially supported by the Polish National Science Centre (grant 2011/01/B/NZ3/00004).

\section{References}

1. Wang N, Wilkin A, Böcking A and Tribukait B: Evaluation of tumor heterogeneity of prostate carcinoma by flow and image DNA cy tometry and histopathological grading. Anal Cell Physiol 20: 49-62, 2000.

2. Singh RK and Talmadge JE: The evolution of diversity within tumors and metastases. In: Selected Aspects of Cancer Progression: Metastasis, Apoptosis and Immune Response. Kaiser HE and Nasir A (eds). Springer Science-Business Media BV, Dordrecht, pp59-90, 2008.

3. Marusyk A and Polyak K: Tumor heterogeneity: causes and consequences. Biochim Biophys Acta 1805: 105-117, 2010.

4. Dirks P: Cancer stem cells. Invitation to a second round. Nature 466: 40-41, 2010.

5. Carter HB and Coffey DS: Cell surface charge in predicting metastatic potential of aspirated cells from the Dunning rat prostate adenocarcinoma model. J Urol 140: 173-175, 1988.

6. Carter HB, Partin AW and Coffey DS: Cell surface charge in predicting metastatic potential in an animal model of prostate cancer: flow cytometric quantification of cell surface charge. J Urol 142: 1338-1341, 1989.

7. Fraser SP, Ding Y, Liu A, Foster CS and Djamgoz MBA: Tetrodotoxin suppress morphological enhancement of the metastatic MAT-LyLu rat prostate cell line. Cell Tissue Res 295: 505-512, 1999.

8. Bironaite D, Nesland JM, Dalen H, Risberg B and Bryne M: $\mathrm{N}$-Glycans influence the in vitro adhesive and invasive behaviour of three metastatic cell lines. Tumor Biol 200: 165-175, 2000.

9. Djamgoz MBA, Mycielska M, Madeja Z, Fraser SP and Korohoda W: Directional movement of rat prostate cancer cells in direct-current electric field. Involvement of voltage gated $\mathrm{Na}^{+}$ channel activity. J Cell Sci 114: 2697-2705, 2001.

10. Pokorna E, Zicha D, Chaloupkova A, Matousková E and Vesely P: Two dynamic morphotypes of sarcoma cells, asymmetric stellate and triangle with leading lamella, are related to malignancy. Folia Biol 49: 33-39, 2003.

11. Sanford KK, Likely GD and Earle WR: The development of variations in transplantability and morphology within a clone of mouse fibroblasts transformed to sarcoma-producing cells in vitro. J Natl Cancer Inst 15: 215-237, 1954.

12. Abercrombie M and Ambrose EJ: The surface properties of cancer cells: a review. Cancer Res 22: 332-345, 1962.

13. Moscona A, Trowell QA and Willmer EN: Methods. (Chap. 2). In: Cell and Tissues in Culture. Vol. 1. Willmer EN (ed). Academic Press, London, pp1-98, 1965.

14. Burger MM: The significance of surface structure changes for growth control under crowded conditions. In: Growth Control in Cell Cultures. Wolstenhome GEW and Knight J (eds). Churchill Livingstone, London, pp45-62, 1971.

15. Wyckoff JB, Segall JE and Condeelis JS: The collection of the motile population of cells from a living tumor. Cancer Res 60: 5401-5404, 2000.

16. Thiery JP: Epithelial-mesenchymal transitions in development and pathogenesis. Curr Opin Cell Biol 15: 740-746, 2003. 
17. Savagner P: The epithelial-mesenchymal transition (EMT) phenomenon. Ann Oncol 21 (Suppl 7): vii89-vii92, 2010.

18. Graham TR, Zhau H, Odera-Marah VA, Osunkoya AO Kimbrok S, Tighiouart M, Liu T, Simons JW and O'Regan RM: Insulin-like growth factor-I-dependent up-regulation of ZEB1 drives epithelial-to-mesenchymal transition in human prostate cancer cells. Cancer Res 68: 2479-2488, 2008.

19. Klarmann GJ, Hurt EM, Matheus LA, Zhang X, Duhagon MA, Mistree T, Thomas SB and Farrar WL: Invasive prostate cancer cells are tumor initiating cells that have a stem cell-like genomic signature. Clin Exp Metastasis 26: 433-446, 2009

20. Tate AW, Lung T, Radhakrishnan A, Lim SD, Lin X and Edlund M: Changes in gap junctional connexin isoforms during prostate cancer progression. Prostate 66: 19-31, 2006.

21. Kanczuga-Koda L, Sulkowski S, Lenczewski A, Koda M, Wincewicz A, Baltaziak $M$ and Sulkowska M: Increased expression of connexins 26 and 43 in lymph node metastases of breast cancer. J Clin Pathol 59: 429-433, 2006.

22. Baritaki S, Chapman A, Yeung K, Spandidos DA, Palladino M and Bonavida B: Inhibition of epithelial to mesenchymal transition in metastatic prostate cancer cells by the novel proteasome inhibitor, NPI-0052: pivotal roles of Snail repression and RKIP induction. Oncogene 28: 3573-3585, 2009.

23. Czyz J, Szpak K and Madeja Z: The role of connexins in prostate cancer promotion and progression. Nat Rev Urol 9: 274-282, 2012.

24. Lentini A, Mattioli P, Nicolini L, Pietrini A, Abbruzzese A and Beninati S: Anti-invasive effects of theophylline on experimental B16-F10 melanoma lung metastasis. Cancer J 10 274-278, 1997.

25. Spangler JG: Bone biology and physiology: implications for novel osteoblastic osteosarcoma treatments. Med Hypotheses 70: 281-286, 2008

26. Holick CN, Smith SG, Giovannucci E and Michaud DS: Coffee, tea, caffeine intake, and risk of adult glioma in three prospective cohort studies. Cancer Epidemiol Biomarkers Prev 19: 39-47, 2010.

27. Wilson KM, Kasperzyk JL, Rider JR, Kenfield S, Van Dam RM, Stampfer MJ, Giovannucci E and Mucci LA: Coffee consumption and prostate risk and progression in the health professionals follow up study. J Natl Cancer Inst 103: 876-884, 2011.

28. Isaacs JT: The R-3327 system of rat prostatic cancers. Urol Oncol 2: 115-116, 1996.
29. Ramaekers FC, Verhagen AP, Isaacs JT, Feitz WF, Moesker O, SchaartG, Schalken JA and Voijs GP: Intermediate filament expression and the progression of prostatic cancer as studied in the Dunning R-3327 rat prostatic carcinoma system. Prostate 14: 323-339, 1989.

30. Erickson CA and Nuccitelli R: Embryonic fibroblast motility and orientation can be influenced by physiological electric fields. J Cell Biol 98: 296-307, 1984

31. Korohoda W and Madeja Z: Contact of sarcoma cells with aligned fibroblasts accelerated their displacement: computer-assisted analysis of tumour cell locomotion in co-culture. Biochem Cell Biol 75: 263-276, 1997.

32. Waligorska A, Wianecka-Skoczen M, Nowak P and Korohoda W: Some difficulties in research into cell motile activity under isotropic conditions. Folia Biol 55: 9-16, 2007.

33. Daniel-Wojcik A, Misztal K, Bechyne I, Sroka J, Miekus K, Madeja Z and Czyz J: Cell motility affects the intensity of gap junctional coupling in prostate carcinoma and melanoma cell populations. Int J Oncol 33: 309-315, 2009.

34. Miekus K, Czernik M, Sroka J, Czyz J and Madeja Z: Contact stimulation of prostate cancer cell migration: the role of gap junctional coupling and migration stimulated by heterotypic cellto-cell contacts in determination of the metastatic phenotype of Dunning rat prostate cancer cells. Biol Cell 97: 893-903, 2005.

35. Burke JM, Skumasz CMB, Irwing PE and McKay BS: Phenotypic heterogeneity of retinoid pigment epithelial cells in vitro and in situ. Exp Eye Res 62: 63-73, 1996.

36. Forrester JA: Microelectrophoresis of normal and polyoma virus transformed hamster kidney fibroblasts. In: Cell Electrophoresis. Ambrose EJ (ed). J. \& A. Churchill Ltd., London, pp115-124, 1965.

37. Korohoda W and Czyz J: Efficacy of the Frame and Hu mathematical model for the quantitative analysis of agents influencing growth of chick embryo fibroblasts. Folia Histochem Cytobiol 32: 113-118, 1994.

38. Prasad KN and Sheppard JR: Inhibitor of cyclic-nucleotide phosphodiesterase tumor cells induce morphological differentiation of mouse neuroblastoma cell culture. Exp Cell Res 73: 436-440, 1972.

39. Weiss L and Subjeck JR: Electrical heterogeneity of the surfaces of Ehrlich ascites tumor cells. Ann NY Acad Sci 238: 352-361, 1974. 